\title{
Photonic Crystal Fibers infiltrated with Ferroelectric Liquid Crystals
}

\author{
Daniel Budaszewski ${ }^{*}$, Tomasz R. Woliński ${ }^{1}$, Morten A. Geday ${ }^{2}$, José M. Otón ${ }^{2}$ \\ ${ }^{l}$ Optics and Photonics Division, Faculty of Physics, Warsaw University of Technology, \\ Koszykowa 75, 00-662 Warszawa, Poland \\ ${ }^{2}$ Departamento de Tecnología Fotónica, ETSI Telecomunicatión, \\ Universidad Politécnica de Madrid, Ciudad Universitaria, Madrid, Spain
}

Received September 16, 2010; accepted September 27, 2010; published September 30, 2010

\begin{abstract}
In the letter we present preliminary results of research on thermal and electrical tunability of photonic crystal fibers (PCF) infiltrated with ferroelectric liquid crystal (FLC) molecules. From this combination we expect new spectral properties to be observed, mainly due to the fast switching time of FLC molecules that can lead to the design of a new kind of optical filters. We have also obtained promising results about the spectral properties of photonic ferroelectric liquid crystal fibers (PFLCF), as well as orientation of FLC molecules inside PCF microholes.
\end{abstract}

Over the last two decades a new type of micro-structured optical fibers known as Photonic Crystal Fibers (PCF) [1] has been investigated by many research groups all over the world. PCFs are made of glass or plastic with a periodic matrix of air micro-holes forming a 2dimensional photonic crystal with defects inside the core region. Depending on the type of a PCF, the defect can be either a solid rod or a hole in the core region breaking the periodic photonic structure. In general, we can distinguish two mechanisms of light propagation in PCF structures. The first one, called the modified total internal reflection (m-TIR), occurs when the refractive index of a core is higher than the effective refractive index of the surrounding cladding made of a photonic structure, so the whole light spectrum is guided in the PCF fiber. The second mechanism called the photonic bandgap effect (PBG) is observed when the refractive index of the core is lower than the effective refractive index of the cladding. In this case only selected wavelengths are guided in the PCF structure.

Light propagation properties of PCFs can be dynamically tuned by filling the micro-holes with substances whose refractive indices can be controlled by external physical fields. Amongst the most suitable substances for infiltrating a PCF structure are liquid crystals (LCs) because of their electro- and thermo-optic properties. Such a hybrid structure combining the micro-structured PCF host with organic guest liquid crystals forms a new class of tunable media called Photonic Liquid Crystal
Fibers (PLCFs) [2]. PLCFs open the door for a diversity of new optical properties (spectral, thermo-optic, electrooptic, polarization etc.). Experiments concerning tuning of nematic liquid crystals inside a PCF were reported for the first time in 2003 [2] and later by other groups [3-7]. It has been successfully shown that thermally-induced switching between two possible guiding mechanisms inside the PLCF is possible. Recently there has been new interest in infiltrating PCFs with chiral nematics [8] or smectic $C^{*}$ liquid crystals which posses ferroelectric properties [9, 10]. By filling the PCF structure with ferroelectric liquid crystals the bandgap position in the transmission spectrum may be tuned with an external electric field. By changing the temperature of the ferroelectric liquid crystal inside a PCF, it can be also possible to tune the spectral position of the bandgaps.

The ferroelectric liquid crystal material used in our research is an experimental mixture MT2_B15 synthesized at the Military University of Technology (Warsaw, Poland), which has a Smectic $C^{*}$ phase at room temperature up to $63^{\circ} \mathrm{C}$.

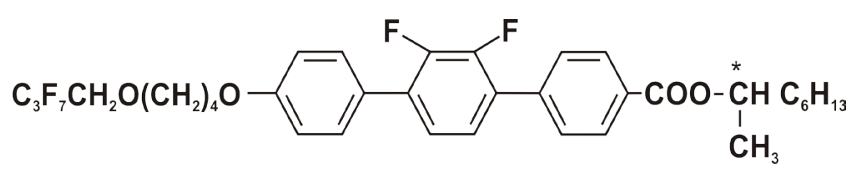

Fig. 1. Chemical structure of MT2_B15 ferroelectric liquid crystal molecule used in the experiments.

All the experiments were performed at room temperature. The molecules of an FLC were infiltrated into an experimental isotropic photonic crystal fiber 070123 (Fig.2) with a fused-silica solid core, manufactured at the Marie Curie Sklodowska University in Lublin, Poland. The diameter of the PCF microholes was $4.1 \mu \mathrm{m}$ with a pitch of $6.5 \mu \mathrm{m}$.

\footnotetext{
*E-mail: danielb@if.pw.edu.pl
} 


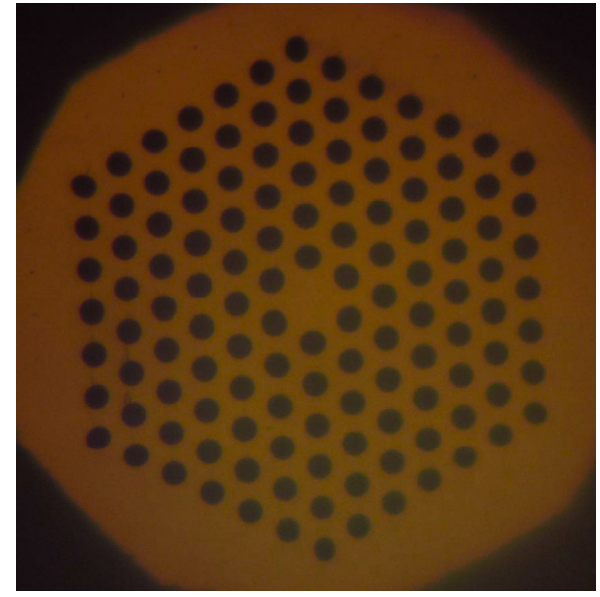

Fig. 2. A cross-section of the 070123 PCF (MCSU, Lublin, Poland).

The infiltration process was performed by dipping one end of the PCF into a container with the FLC material heated up to $150^{\circ} \mathrm{C}$. Due to capillary forces, the molecules of the FLC got drawn into the PCF structure.

In Fig 3 we present a PCF fiber partially filled with molecules of the MT2_B15 FLC observed under a microscope with crossed polarizers.

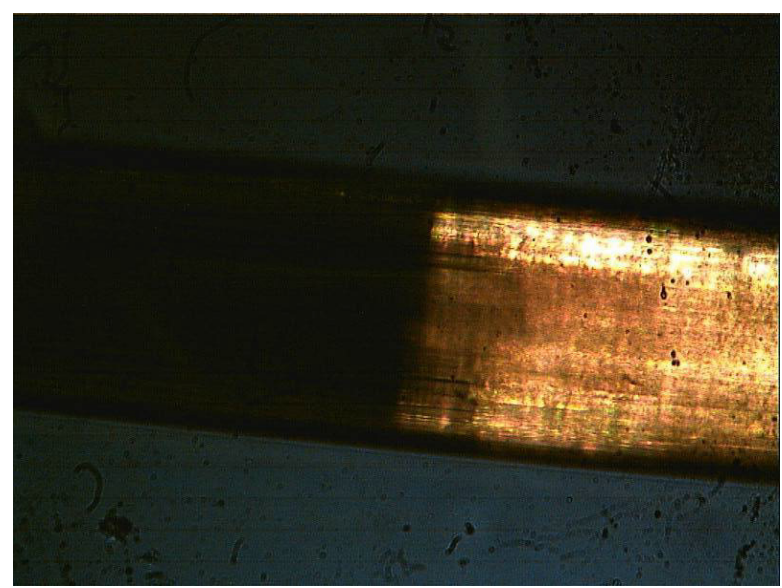

Fig. 3. Photonic Crystal Fiber with filled and non-filled part under a microscope with crossed polarizers.

According to our observations of the PFLCF structure under a polarization microscope we noticed that the orientation of the molecules in the PCF structure appears to be random and that an external electric field can reorient the FLC molecules. In Fig. 4 we present the schematic diagram of an experimental setup for investigating the orientation of FLC molecules under the influence of an external electric field. As a light source we used a broadband supercontinuum light source NKT Photonics. The signal at the output of the PFLCF was registered by the fiber optic spectrometer Ocean Optics USB4000.

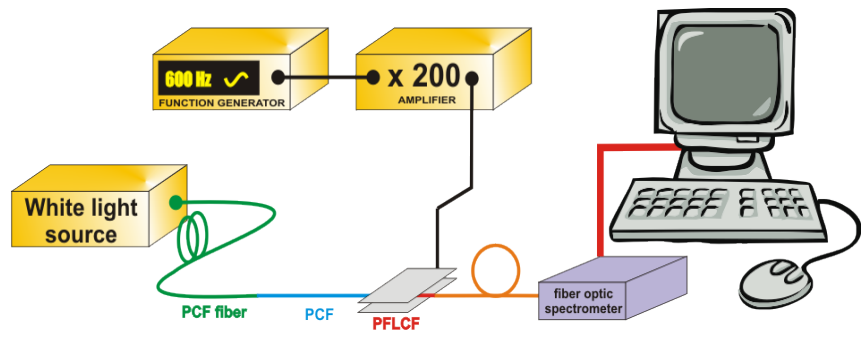

Fig. 4. Schematic diagram of an experimental setup for investigating the tunability of an FLC under an external electric field.

We applied a $600 \mathrm{~Hz}$ sinusoidal external electrical field, which was amplified to generate peak voltages varying from 0 to $500 \mathrm{~V}$ in order to switch the FLC molecules inside the PCF microholes (Fig. 5).

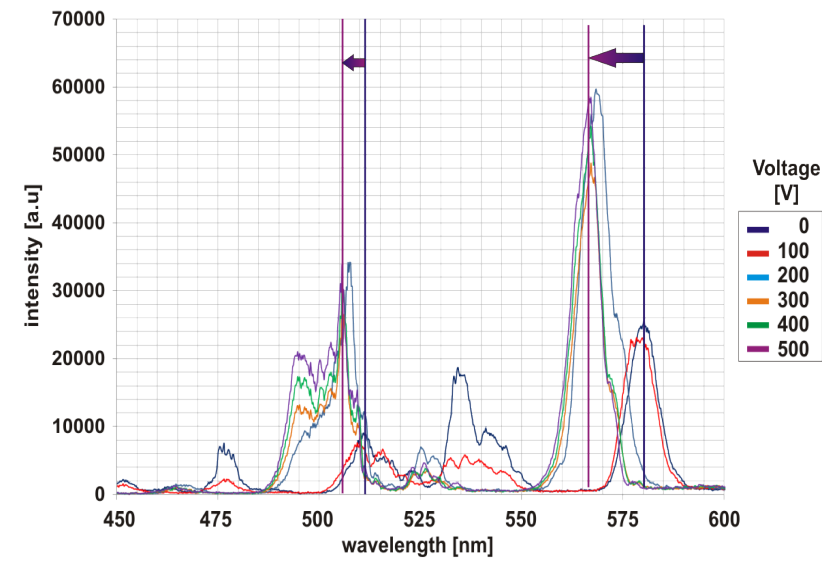

Fig. 5. Transmission spectrum of a PFLCF under the influence of an external electric field.

As shown in Fig. 5, the photonic bandgaps in the transmission spectrum are observed. By increasing the voltage, a blue-shift effect in two regions of the spectrum was noticed. The shift of one bandgap was $5 \mathrm{~nm}$ from $510 \mathrm{~nm}(0 \mathrm{~V})$ to $505 \mathrm{~nm}(500 \mathrm{~V})$, and $14 \mathrm{~nm}$ for the second bandgap, from $579 \mathrm{~nm}(0 \mathrm{~V})$ to $566 \mathrm{~nm}(500 \mathrm{~V})$.

It should be pointed out that in a case of electric tuning of PFLCF, the observed blue-shift of bandgap positions seems to be a switch-like shift. Up to $100 \mathrm{~V}$ between electrodes no significant shift in the bandgap spectrum has been observed. After exceeding $200 \mathrm{~V}$ a rapid blueshift has been noticed. By increasing the voltage from 
$200 \mathrm{~V}$ up to $500 \mathrm{~V}$ between electrodes once more no significant shift has been noticed.

In the next step we investigated thermal tuning of the PFLCF transmission spectrum. We replaced the electrodes with the Peltiere module to heat up the filled part of a PFLCF. The temperature of the PFLCF sample was varied between 30 and $55^{\circ} \mathrm{C}$ to investigate the tuning properties of photonic bandgaps in the smectic $\mathrm{C}^{*}$ phase as a function of temperature (Fig. 6)

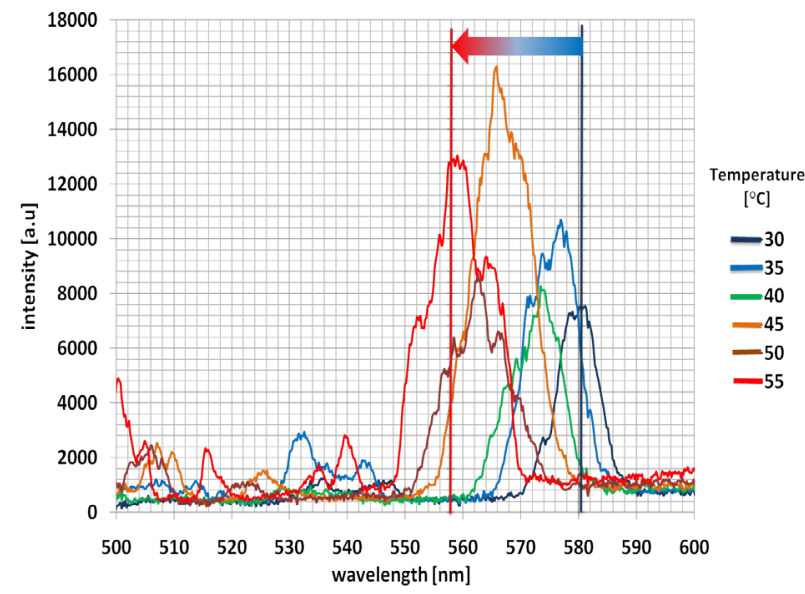

Fig. 6. Transmission spectrum of a PFLCF under the influence of temperature.

By increasing the temperature of the sample, we also noticed the blue-shift effect in the transmission spectrum. The observed shift was $\sim 20 \mathrm{~nm}$ from $580 \mathrm{~nm}$ (in $30^{\circ} \mathrm{C}$ ) to $560 \mathrm{~nm}$ (in $55^{\circ} \mathrm{C}$ ). Unlike to electric tuning of PFLCF the bandgap position changes have rather a continuous character.
To conclude, ferroelectric liquid crystals can be considered as good candidates for a new kind of optical filters and attenuators mainly due to their fast response to an external electric field.

This work was supported by the Programme S2009/ESP-1781 FACTOTEM 2 and partially supported by the Polish grants: MSHE-N517056535 and the FNP Master programme. The authors would like to thank Prof. Roman Dąbrowski for the FLC mixtures, and the late Dr. Jan Wójcik for the PCF samples.

\section{References}

[1] P. St. J. Russell, J. Lightwave Technol. 24, 4729-4749 (2006).

[2] T. Larsen, A. Bjarklev, D. Hermann, and J. Broeng, Opt. Express 11, 2589-2596 (2003).

[3] T. R. Wolinski, K. Szaniawska, K. Bondarczuk, P. Lesiak, A.W. Domanski, R. Dabrowski, E. Nowinowski-Kruszelnicki, J. Wojcik, Opto-Electronics Rev. 13(2), 59-64 (2005).

[4] S. Ertman, T. Nasiłowski, T.R.Woliński, H. Thienpont, Phot. Lett. of Poland 1(1), 13-15 (2009).

[5] M. Tefelska, M. Chychłowski, T.R. Woliński, R. Dąbrowski, J. Wójcik, Phot. Lett. of Poland 1(2), 97-99 (2009).

[6] M.W. Haakestad, T. T. Alkeskjold, M. D. Nielsen, L. Scolari, J. Riishede, H. E. Engan, and A. Bjarklev, IEEE Photon. Technol. Lett. 17, 819-821 (2005).

[7] Q. Lu and S. T.Wu, Appl. Phys. Lett. 85, 2181-2183 (2005).

[8] M. Tefelska, T.R. Woliński, R. Dąbrowski, J. Wójcik, Phot. Lett. of Poland 2(1), 28-30 (2010)

[9] S. Mathews, Y. Semenova, G. Rajan, G. Farrell, Proc. of SPIE 7316, 73161E-1 - 73161E-6 (2009).

[10] S. Mathews, Y. Semenova, G. Farrell, Electr. Lett. 45(12) 617 - 618 (2009). 